BULL. AUSTRAL. MATH. SOC.

VOL. 30 (1984), 11-18.

$(47 \mathrm{~B} 05,47 \mathrm{~B} 20)$

\title{
ON THE STRUCTURE OF POLYNOMIALLY NORMAL OPERATORS
}

FUAD KITTANEH

We present some results concerning the structure of polynomially normal operators. It is shown, among other things, that if $T^{n}$ is normal for some $n>1$, then $T$ is quasi-similar to a direct sum of a normal operator and a compact operator and if $p(T)$ is normal with $T$ essentially normal, then $T$ can be written as the sum of a normal operator and a compact operator. Utilizing the direct integral theory of operators we finally show that if $p(T)$ is normal and $T^{*} T$ commutes with $T+T^{*}$, then $T$ must be normal.

\section{Introduction}

Let $\mathrm{H}$ be a separable, infinite dimensional complex Hilbert space, and let $B(H)$ denote the algebra of all bounded linear operators on $H$. An operator $T \in B(H)$ is called normal if $T T^{*}=T * T$ where $T^{*}$ denotes the adjoint of $T$. It is clear that if $T$ is normal then any polynomial of $T$ is also normal. However, the converse is not true. To see this take any non-normal $T$ such that $T^{2}=0$. Roots of normal operators have been extensively studied and many beautiful results have been obtained (see [5] and its references).

This paper has two purposes, the first is to give some structure

Received 10 January 1984. I would like to thank my thesis advisor, Professor Joseph G. Stampfli, for his suggestions.

Copyright Clearance Centre, Inc. Serial-fee code: 00049727/84, $\$ \mathrm{~A} 2.00+0.00$ 
theorems for power normal operators, for example, we will show that if $T^{n}$ is normal for some $n>1$, then $T$ is quasi-similar to a direct sum of a normal operator and a compact operator. Using a result of F. Gilfeather and some of the B-D-F results we will show that if $P(T)$ is normal for some non-zero polynomial $P$ and $T$ is essentially normal, then $T$ can be written as a sum of a normal operator and a compact operator. The second purpose is to give some sufficient conditions to insure the normality of $T$ whenever $P(T)$ is normal. As an example we will utilize the direct integral theory of operators to show that if $P(T)$ is normal and $T * T$ commutes with $T+T^{*}$, then $T$ is normal.

\section{Structure Theorems}

In this section, we shall present several structure theorems and representations for $T$ whenever some polynomial of $T$ is normal. These results depend heavily upon the beautiful representation theorem of H. Radjavi and P. Rosenthal [8].

our first result can be stated as follows:

THEOREM 1. Let $T \in B(H)$ be such that $T^{2}$ and $P(T)$ are normal.

(a) If $P(Z)=a_{0}+a_{1} z+a_{2} z^{2}+\ldots+a_{n} z^{n}$ where $n>2$ and at least two odd powers coppear, then $T=V \oplus S$ with $V$ normal and $S$ algebraic.

(b) If $p(z)=a_{0}+a_{1} z+a_{2} z^{2}+\ldots+a_{n} z^{n}$ where $n>2$ and one and only one odd power appears, then $T=V \oplus S$ with $V$ normal and $S$ nilpotent of index 2 .

Proof. Since $\mathrm{T}^{2}$ is normal, by [8] $\mathrm{T}$ can be written as

$$
T=\left[\begin{array}{rrr}
A & 0 & 0 \\
0 & B & C \\
0 & 0 & -B
\end{array}\right]
$$

where $A, B$ are normal, $C \geq 0, C$ is one to one and $B C=C B$. Furthermore, $B$ can be chosen so that $\sigma(B)$ lies in the closed upper half-plane. 
Let $p(z)=a_{0}+a_{1} z+a_{2} z^{2}+\ldots+a_{n} z^{n} ;$ then

$$
p(T)=\left[\begin{array}{ccc}
p(A) & 0 & 0 \\
0 & p(B) & x \\
0 & 0 & p(-B)
\end{array}\right]
$$

for some $x$. If $k$ is the only odd integer such that $a_{k} \neq 0$, then it is not hard to see that $x=a_{k} B^{k-1} C . \quad P(T)$ being normal implies

$$
p\left(B^{*}\right) p(B)=p(B) p\left(B^{*}\right)+\left|a_{k}\right|^{2} C^{2} B^{k-1} B^{*}{ }^{k-1}=0 \text {. }
$$

since $C$ is one to one, $B^{k-1}=0$. Thus $B=0$ (the only nilpotent normal operator is the zero operator).

Let $\mathrm{V}=\mathrm{A}$ and $\mathrm{S}=\left[\begin{array}{ll}0 & \mathrm{C} \\ & \\ 0 & 0\end{array}\right]$. Hence $\mathrm{T}=\mathrm{V} \oplus \mathrm{S}$ with $\mathrm{V}$ normal and $\mathrm{s}^{2}=0$, which proves (b). The proof of (a) can be completed similarly.

COROLLARY 1. If $T \in B(H), T^{2}$ and $a_{0}+a_{1} T+a_{2} T^{2}+a_{1} T^{3}+a_{4} T^{4}$ are normal, then $T$ is similar to a normal operator.

Proof. Using the same notation as in the proof of Theorem 1, we have

$$
p(T)=\left[\begin{array}{ccc}
p(A) & 0 & 0 \\
0 & p(B) & x \\
0 & 0 & p(-B)
\end{array}\right] \text {, }
$$

where $p(z)=a_{0}+a_{1} z+a_{2} z^{2}+a_{1} z^{3}+a_{4} z^{4}$, and $x=a_{1} c+a_{1} B C^{2}$.

since $p(T)$ is normal, it follows that $x=0$. $C$ being one to one implies that $I+B^{2}=0$. But $\sigma(B)$ being contained in the closed upper half-plane implies that $B+i$ is invertible, and so $B=i$. Hence

$$
T=\left[\begin{array}{rrr}
A & 0 & 0 \\
0 & i & C \\
0 & 0 & -i
\end{array}\right]
$$

which is similar to the normal operator

$$
\left[\begin{array}{rrr}
A & 0 & 0 \\
0 & i & 0 \\
0 & 0 & -i
\end{array}\right]
$$


The similarity is implemented by the invertible operator

$$
\left[\begin{array}{ccc}
1 & 0 & 0 \\
0 & 1 & c / 2 i \\
0 & 0 & 1
\end{array}\right] .
$$

COROLLARY 2. Let $T$ be as in Theorem $1(b)$ and $T$ be essentially normal (that is, $T^{*} T-T T^{*} \in K(H)$ the ideal of compact operators), then $T$ is the direct sum of a normal operator and a compact operator.

Proof. Since $\mathrm{T}=\mathrm{V} \oplus \mathrm{S}$, with $\mathrm{V}$ normal and $\mathrm{S}^{2}=0$, $T^{*} T-T^{*} \in K(H)$ implies that $S^{*} S-S S^{*} \in K(H)$. Therefore $\pi(S)$ is a normal nilpotent element of the Calkin algebra $B(H) / K(H)$, with $\pi$ being the canonical map of $B(H)$ onto $B(H) / K(H)$. Hence $\pi(S)=0$, and so $S$ is compact.

An operator $T \in B(H)$ is said to be quasidiagonal, or quasitriangular if there exists a sequence $\left\{\mathrm{p}_{n}\right\}_{n=0}^{\infty}$ of finite-rank projections converging strongly to 1 such that $\left\|\mathrm{P}_{n} \mathrm{~T}-\mathrm{TP}_{n}\right\| \rightarrow 0$ or $\left\|\mathrm{P}_{n}{ }^{\mathrm{TP}}{ }_{n}-{ }^{T P}{ }_{n}\right\| \rightarrow 0$, respectively (see [6] and [7]). It is known that a normal operator is both a quasidiagonal and a quasitriangular operator [6]. Now we give the following generalization of this result.

COROLLARY 3. Let $T$ be as in Theorem 1(b), then $T$ is quasidiagonal.

Proof. We have $\mathrm{T}=\mathrm{V} \oplus \mathrm{S}$ with $\mathrm{V}$ normal and $\mathrm{s}^{2}=0$. It is known that every normal operator is quasidiagonal, and by $[10]$ every nilpotent of index 2 is quasidiagonal. Since the direct sum of two quasidiagonal operators is quasidiagonal [6], the result follows.

From the quasitriangularity case we require the following remarkable result due to $F$. Gilfeather.

THEOREM 2. Let $T \in B(H)$ be such that $p(T)$ is normal for some polynomial $p$. Then there exist reducing subspaces $\left\{H_{n}\right\}_{n=0}^{\infty}$ for $T$ such that $H=\bigoplus_{n=0}^{\infty} H_{n}, T_{0}=T \mid H_{0}$ is algebraic, and $T_{n}=T \mid H_{n}$ is simizar to a normal operator.

Proof. See [5]. 
COROLLARY 4. If $T \in B(H)$ is polynomially normal, then $T$ is quasitrianguzar.

Proof. In the theory of quasitriangular operators it is known that (see [7]) an operator with countable spectrum is quasitriangular, an operator similar to a quasitriangular one is quasitriangular and any countable direct sum of quasitriangular operators is quasitriangular. In view of these properties, the result now follows by Theorem 2 .

Remark. Applying the corollary to $T^{*}$ as well, we conclude that $T$ is biquasitriangular, that is, $T$ and $T^{*}$ are quasitriangular.

COROLLARY 5. If $T$ is a polynomially normal operator, then $T$ is a norm-limit of algebraic operators.

Proof. The result follows from a remarkable characterization given by Voiculescu [12] which asserts that the set of biquasitriangular operators coincides with the norm-closure of the set of algebraic operators.

COROLLARY 6. If $T$ is polynomially normal and essentially normal, then $T$ can be written as the swo of a normal operator and a compact one, hence $T$ is quasidiagonal.

Proof. The corollary follows from a result of [1] which states that if $T$ is essentially normal such that both $T$ and $T^{*}$ are quasitriangular, then $T$ is normal plus compact.

Corollary 6 admits the following generalization.

THEOREM 3. If $T$ is polynomially normal and essentially hyponormal, then $T$ can be written as the sum of a normal operator and $a$ compact one.

Proof. Suppose that $p(T)$ is normal, for some polynomial $p$. Since $\pi(p(T))=p(\pi(T))$, it follows that $p(\pi(T))$ is normal in $B(H) / K(H)$. Since $B(H) / K(H)$ is a $C^{*}$-algebra, there exist a Hilbert space $\mathrm{H}_{0}$ and an isometric *-isomorphism $v$ of $\mathrm{B}(\mathrm{H}) / \mathrm{K}(\mathrm{H})$ into $\mathrm{B}\left(\mathrm{H}_{0}\right)$, (see [4]). Since $p(\nu \circ \pi(T))=\nu(p(\pi(T)))$, and $p(\pi(T))$ is normal, then $v \circ \pi(T)$ is polynomially normal and hyponormal operator in $B\left(H_{0}\right)$. Thus $v \circ \pi(T)$ is normal [11], and so $\pi(T)$ is normal. The result now follows by corollary 6 . 
Two operators $S$ and $T$ in $B(H)$ are said to be quasi-similar if there exist two operators $X$ and $Y$ in $B(H)$, which are one to one and of dense range, such that $S X=X T$ and $Y S=T Y$. The importance of quasi-similarity for the invariant subspace problem lies in the fact that for two quasi-similar operators $S$ and $T$, if one of them has a proper hyperinvariant subspace, so does the other one. The following well-known lemma [7] enables us to prove that a power normal operator is quasisimilar to a direct sum of a normal operator and a compact operator.

LEMMA. Suppose $\left\{H_{n}\right\}_{n=0}^{\infty}$ is a sequence of Hilbert spaces and for each $n, S_{n} A_{n} S_{n}^{-1}=B_{n}$, where $A_{n}, B_{n} \in B\left(H_{n}\right)$, and $S_{n}$ is an invertible operator in $B\left(H_{n}\right)$. Then the operators $A=\bigoplus_{n=0}^{\infty} A_{n}$ and $B=\bigoplus_{n=0}^{\infty} B_{n}$ acting on the Hizbert space $H=\bigoplus_{n=0}^{\infty} H_{n}$ are quasi-similar.

THEOREM 4. Let $T \in B(H)$ be such that $T^{n}$ is normal for some $n>1$. Then $T$ is quasi-similar to a direct sum of a normal operator and a compact one.

Proof. By Theorem 2, $T=\bigoplus_{n=0}^{\infty} T_{n}$, where $T_{0}$ is nilpotent and $T_{n}$ is similar to a normal operator $\mathrm{N}_{\mathrm{n}}$. It is known that every nilpotent operator is quasi-similar to a compact operator (see [7]). Thus $\mathbf{T}_{0}$ is quasi-similar to some compact operator $\mathrm{K}$ in $\mathrm{B}\left(\mathrm{H}_{0}\right)$. By the Lemma $\bigoplus_{n=1}^{\infty} T_{n}$ is quasi-similar to the normal operator $N=\bigoplus_{n=1}^{\infty} N$ on the Hilbert space $\bigoplus_{n=1}^{\infty} H_{n}$. Therefore $T$ is quasi-similar to $N \oplus K$ as required.

2. Conditions implying the normality of a polynomially normal operator

It has been shown by stampfli [11] that a power normal operator which is hyponormal must be normal. The direct integral representation [9] enables us to give a different proof for this fact and to establish an analogous result for the class of operators considered by Campbell in [2]; where a special case when $p(z)=z^{2}$ was proved. 
THEOREM 5. If $T$ is polynomially normal and $T^{*} T$ commutes with $T+T^{*}$, then $T$ is normal.

Proof. Assume that $p(T)$ is normal for some polynomial $p$. Let $A$ be the abelian von Neumann algebra generated by $p(T)$. Then the underlying Hilbert space $\mathrm{H}$ can be written as a direct integral $H=\int^{\oplus} H(x) d \mu(x)$ such that each operator in $A$ is diagonal and each operator in $A^{\prime}$, the commutant of $A$, is decomposable relative to this representation. Since $T \in A^{\prime}$, we have $T=\int^{\oplus} T(x) d \mu(x)$ for almost every $x$. Since $p(T)$ can be expressed as $P(T)=\int^{\oplus} f(x) d \mu(x)$ for some $f \in L^{\infty}(\mu)$, it follows that $p(T(x))=f(x)$ for almost all $x$ and so $T(x)$ is algebraic. From [2] and [3] one can conclude that if $T \star T$ commutes with $T+T^{*}$ and $T$ has a countable spectrum, then $T$ is normal. Since for almost all $x, T(x)$ has a finite spectrum and $T *(x) T(x)$ commutes with $T(x)+T^{*}(x)$, we conclude that almost every $T(x)$ is normal. Hence $T$ is normal and the proof is complete.

THEOREM 6. If $T$ is a polynomially normal operator which is hyponormal, then $T$ is normal.

Proof. Since it is known [11] that a hyponormal operator whose spectrum is countable must be normal, the proof can be completed as that of Theorem 5 .

\section{References}

[1] L.G. Brown, R.G. Douglas, and P.A. Fillmore, Unitary equivalence modulo the compact operators and extensions of $\mathrm{C}^{*}$-algebras, Proc. Conf. Operator Theory, Lecture Notes in Math., 345 (1973), 58-128.

[2] S.L. Campbell, "Linear operators for which $T^{\star} T$ and $T+T *$ commute", Pacific J. Math., 61 (1975), 53-57.

[3] S.L. Campbell and R. Gollar, "Spectral properties of linear operators for which $\mathrm{T}^{\star T} \mathrm{~T}$ and $\mathrm{T}+\mathrm{T}^{*}$ commute", Proc. Amer. Math. Soc., $60(1976)$, 197-202.

[4] R.G. Douglas, Banach Algebra Techniques in Operator Theory, (Academic Press, New York and London, 1972). 
[5] F. Gilfeather, "Operator valued roots of abelian analytic functions", Pacific J. Math. , 55(1974), 127-148.

[6] P.R. Halmos, "Ten problems in Hilbert space", BulZ. Amer. Math. Soc. , $76(1970), 887-933$.

[7] C. Pearcy, some recent developments in operator theory, (Lecture Notes, No. 36, Amer. Math. Soc., Providence, R.I., 1978).

[8] H. Radjavi and P. Rosenthal, "On roots of normal operators", J. Math. Anal. App Z., 34(1971), 653-664.

[9] J. Schwartz, W*-algebras, (Gordon and Breach, New York, 1967).

[10] R.A. Smucker, Quasidiagonal and quasitriangular operators. PhD thesis, Indiana University, 1973.

[11] J.G. Stampfli, "Hyponormal operators", Pacific J. Math. 12(1962), 1453-1458.

[12] D. Voiculescu, "Norm limits of algebraic operators", Rev. Rowmaine Math. Pures Appl., 19(1974), 371-378.

Department of Mathematics,

United Arab Emirates University,

PO Box 15551,

Al-Ain, United Arab Emirates 